\title{
Cognitive and behavioural approaches to medication adherence
}

\author{
Jan Scott
}

\begin{abstract}
"The desire to take medication is perhaps the greatest feature which distinguishes man from animals." (Sir William Osler)
\end{abstract}

Medication non-adherence is a major obstacle to translating treatment efficacy in research settings into effectiveness in clinical practice (Dickson \& Kendall, 1986; Scott, 1995). Randomised controlled trials indicate that brief interventions such as cognitivebehavioural educational packages for depression, cognitive therapy for lithium clinic attenders and compliance therapy for people with schizophrenia may be beneficial (Cochran, 1984; Katon et al, 1995; Kemp et al, 1996). However, clinical psychiatry has been surprisingly slow to investigate individual risk factors for medication non-adherence or to use strategies to enhance adherence that have been used extensively in other chronic illness populations.

In this article.I suggest that taking a systematic approach to the examination of a patient's healthrelated beliefs, the factors that influence those beliefs, and the prompts that lead people to engage in certain behaviours, can improve our clinical ability to identify and intervene in cases of non-adherence. Examples will be drawn primarily from work with patients with affective disorders, but the same principles and techniques can be applied to other patient populations.

\section{Definition and prevalence of adherence}

Adherence may be defined as the extent to which an individual engages in recommended health behaviours (Wright, 1993). Medication adherence is important in its own right, but it is also a proxy measure of other adherence behaviours such as attending clinic appointments, reducing intake of illicit drugs or alcohol, or engaging in a more healthy lifestyle.

Adams \& Scott (1999) report that less than $50 \%$ of hospital-treated patients with severe mental disorders who were receiving prophylactic medication for at least two years were fully adherent to the prescribed treatment. This prevalence is unchanged for two decades despite the introduction of a vast number of new drugs with different sideeffect profiles (Haynes et al, 1979; further details available from the author upon request). As such, non-adherence represents a serious public health problem. Twelve-month relapse rates for schizophrenia are $55 \%$ in those who do not take antipsychotics compared to only $14 \%$ in those who adhere to medication. Kecket al (1998) found that $60 \%$ of patients admitted with mania had failed to adhere to medication in the month prior to hospitalisation. In unipolar disorders, the one-year relapse rates are $80 \%$ in patients not taking antidepressants and 30\% for those who adhere. Despite these figures, research demonstrates that about $10 \%$ of patients with depression do not cash their prescription, and $40-60 \%$ stop their medication within three months of commencing treatment (Myers \& Branthwaite, 1992; Lin et al, 1995).

\section{Predicting non-adherence}

Until recently, psychiatrists have paid scant attention to the problem of medication non-

Jan Scott has recently moved from Newcastle to take up the post of Chair and Head of the Department of Psychiatry at the University of Glasgow (Department of Psychological Medicine, University of Glasgow, Gartnavel Royal Hospital, Glasgow G12 OXH). She is the principal investigator for the MRC-funded multi-centre trial of cognitive therapy for bipolar disorders. Her work on medication adherence and bipolar disorders is supported by a legacy from the Searle family, donations from Mrs E. Leighton and The Stanley Foundation. 
adherence. This is unfortunate, as research suggests that mental health professionals only predict with $50 \%$ accuracy which patients are adherent. Early studies of factors associated with non-adherence were overly focused on basic characteristics of the patient sample or the mechanics of the treatment regime (multiple doses of many different drugs with particular side-effect profiles). It is true that nonadherence in psychiatric populations may be associated with demographic factors such as age or gender, with illness factors such as lack of insight, with medication factors such as complexity of the treatment regime or side-effects, or with clinical factors such as the doctor-patient relationship (Goodwin \& Jamison, 1990; Scott, 1995; Buchanan, 1996). However, evidence is equivocal as to which of these factors or combinations of factors is most critical.

Previous studies largely failed to take into account that adherence is not necessarily an 'all or nothing' phenomenon. It may be partial, cyclical or involve alterations in dosage rather than the timing of drug ingestion. Most importantly, the data do not help clinicians to identify which individual from within an 'at risk' group will become non-adherent. Many people report that they simply forget to take their medication. However, some patients with mild sideeffects fail to take medication that they know can ameliorate distressing symptoms, whereas others with severe and troublesome side-effects persist with treatment regimes that are only partially beneficial. Clinicians need to develop a more systematic approach to identifying and understanding which individuals are at risk of non-adherence so that they may appropriately target interventions to enhance adherence.

\section{Identifying individuals at risk of non-adherence}

Researchers will rightly argue that some of the discrepancies between studies of factors predicting non-adherence result from different methods of measuring adherence. Although monitoring of plasma levels of medication is probably the most reliable approach to assessing adherence, it is not always feasible and, even when available, it is not foolproof. In practice, Stephenson et al (1993) demonstrated that a simple question "Do you have difficulties taking your medication as prescribed?" has a specificity of $90 \%$. Although the sensitivity of this question is only $50 \%$, it is important to emphasise that creating an environment where patients can admit to and then discuss non-adherence is probably more important than access to electronic pill bottles or plasma-monitoring systems.
The available evidence highlights that clinicians are dealing with at least two sub-populations of non-adherers. These can usefully be classified as unintentional and intentional non-adherers (Horne, 1997). Much of the earlier research focused on subjects at risk of unintentional non-adherence. These individuals intend to adhere to medication, but are sometimes unable to understand or manage the treatment regime prescribed. Less is known about individuals at risk of intentional non-adherence, but it is important that we explore underlying factors that prompt the decision not to take the treatment offered as instructed. Weintraub (1990) refers to this group as "intelligent non-adherers". He emphasises that from the patient's perspective, not taking medication is a rational decision based on his or her cognitive representation of the illness. As shown in Box 1, this comprises of five key components (identity, cause, time-line, consequences and cure). Although the content of the cognitive representations varies across different demographic and illness groups and is influenced by individual and cultural factors, its structure appears to remain constant (Skelton \& Croyle, 1991).

\section{Primary prevention of non-adherence}

An important goal of clinical care is the prevention of non-adherence. The model described above highlights that it may be more useful for a psychiatrist initially to explore the patient's cognitive representation of his or her illness, rather than to launch into a detailed exposition of the nature of the disorder and the proposed treatment regime. This provides an opportunity to explore the individual's ideas, misconceptions and appraisals of the illness, and the potential treatment options. Adherence is most likely to occur if there is coherence between patients' abstract ideas about their illness, their concrete experience of the symptoms and the psychiatrist's instructions (Horne, 1997). Before the patient leaves the first consultation, the clinician

Box 1. Cognitive representation of illness

Identity What is it?

Cause What caused it?

Time-line How long will it last?

Consequences How will it/has it affected me?

Cure Can it be controlled or cured? 
needs to establish whether, from the patient's perspective, the treatment regime is acceptable, understandable and manageable.

The patient's cognitive representation of his or her illness and appraisal of the coping strategy employed (medication) needs to be re-assessed at regular intervals. Continued adherence is only likely if the coherence between the abstract and concrete aspects of the patient's illness representation is maintained and if the clinician's advice makes sense to the patient in the light of his or her own experiences and representations (Horne, 1997).

\section{A framework for intervening in non-adherence}

When working with patients who are partially or totally non-adherent it is useful to have a framework to describe the specific factors that are influencing an individual's engagement with treatment. A number of models have been proposed, but one that is useful in this context is the 'health beliefs model' (HBM) of Becker \& Maimon (1975).

The HBM assumes that four main beliefs contribute to the likelihood of individuals adhering to their prescribed medication (see Fig. 1). The beliefs relate to:

- perceived benefits of adherence (e.g. possibility of being symptom-free)

- perceived barriers to adherence (e.g. stigma or problems with side-effects)

- perceived susceptibility to illness (e.g. a belief that they are likely to experience a relapse)

- perceived severity of the outcome (e.g. a belief that relapse would have negative consequences)

It is proposed that individuals are more likely to adhere to medication if the perceived threat of the
Modifying factors

Demographic characteristics, e.g. social support, views of significant others

Psychological characteristics, e.g. personality
Individual beliefs about disorder Perceived susceptibility

Perceived severity

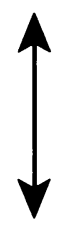

Perceived benefits > perceived barriers e.g. side-effects
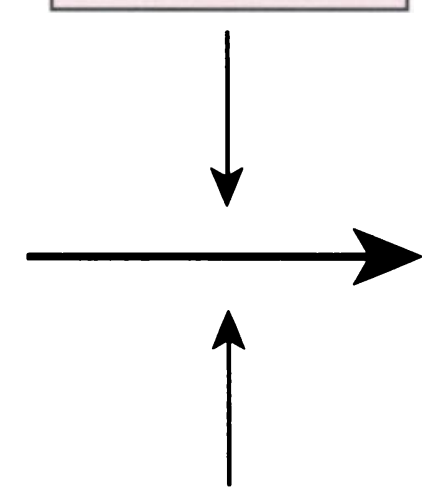

Likelihood of engaging in health behaviours

e.g. medication adherence, clinic attendance, reduced alcohol intake
Cues to action

Internal triggers, e.g. relapse symptoms

External triggers, e.g. advice of others (including doctors), illness in significant other 
illness (susceptibility and severity) is high and the perceived benefits of treatment exceed the perceived barriers. Beliefs associated with adherence may be specific to the illness or the treatment (e.g. doctors do more harm than good), but many represent the general rules or assumptions that operate across the whole spectrum of an individual's life (e.g. I must be in control; I must do everything perfectly).

The beliefs described are influenced by a number of modifying factors (Horne \& Weinman, 1998) such as:

- personality attributes (e.g. dysfunctional attitudes and health locus of control)

- influence of significant others (e.g. family and mental health professionals)

- cultural beliefs and context

- general health motivations

- general orientation towards medicine

The model also states that individuals need a prompt (a reminder either of the threat of the illness or the action that must be taken against it), before they will engage in health-related behaviours (Weinstein, 1988). These 'cues to action' may be internal, such as recognition of prodromal symptoms. Alternatively, the cues may be external, such as statements made by others, or media references to illness or medication.

\section{Cognitive and behavioural strategies}

\section{General approach}

Given the $50 \%$ prevalence of non-adherence, clinicians should assume that maintaining adherence may prove problematic to all patients at some time. The critical factor for successful management of adherence is creating an atmosphere where a nonadherent or potentially non-adherent patient does not feel disapproved of and so is able to talk honestly about his or her concerns about drug treatments and pattern of adherence.

\section{Assessment of health beliefs}

Discussion of individuals' cognitions about mental disorder and fears about treatment is a vital component of the assessment process (Goodwin \& Jamison, 1990). The clinician needs to have a clear picture of the patient's cognitive representation of his or her illness (i.e. his or her answers to the questions in Box 1). With regard to the HBM, the clinician should know about the patient's perceptions of threat (the combination of susceptibility and severity), benefits and barriers to treatment, relevant modifying factors and any cues that prompt individuals to take their medication.

It is important to retain an open mind and not to assume that you know the answers to any of the questions. For example, Adams \& Scott (1999) demonstrated that, when all components of the $\mathrm{HBM}$ were assessed, the desire to be in control of one's life was a significant barrier to adherence but medication side-effects were not.

\section{Analysis of pattern of non-adherence}

If patients acknowledge partial or total nonadherence, it is useful to try to decide whether this is unintentional or intentional. Unintentional nonadherers tend to identify a higher number of perceived barriers to treatment. Most of these are practical rather than psychological. Intentional non-adherers often demonstrate more ambivalence about the perceived threat of the disorder and are probably less likely to acknowledge their nonadherence without prompting. In practice, it is likely that both groups will benefit from the behavioural interventions outlined in Box 2, but that the cognitive techniques will have a more obvious role with intentional non-adherers.

\section{Interventions for unintential non-adherence}

The primary goal with unintentional non-adherers is to enhance cues to action and to minimise any real or perceived barriers to adherence. The key interventions are identified in Box 2.

At a practical level, highly complex treatment regimes can increase the risk of non-adherence, so a simple schedule should be negotiated whenever possible (Goodwin \& Jamison, 1990). This approach may be reinforced by writing the essential details of the regime, situations or symptoms (cues) that may prompt changes in medication doses and recommended action in a crisis on a small card ('flashcard') that can be carried in a pocket or wallet.

A further strategy is the use of behavioural prompts, such as notes stuck in a prominent place that is visited daily (such as a bathroom mirror). 'Pairing' tablet-taking with a routine daily activity (e.g. listening to the morning news) may also aid adherence (Scott \& Wright, 1997). Cognitive rehearsal, where patients visualise each step in their medication routine, may be useful in exposing barriers to adherence. Planning ahead and rehearsing 
coping strategies for novel situations is also beneficial. Other members of the patient's family may be asked to offer reinforcement. However, caution is required and this approach should only be employed where relationships are stable and the illness and its treatment are not a battleground.

The benefits of each strategy can be monitored through keeping a simple medication diary. One or two techniques may be sufficient to enhance adherence. Homework assignments monitoring adherence with the prescribed treatment, its benefits (symptom reduction) and any troublesome sideeffects can then be used to foster further discussion. If patients report problems with side-effects, it is better to initiate discussions on management by asking them to identify the ones they know about or which are of particular concern, rather than running through a check-list of medication side-effects (Goodwin \& Jamison, 1990). Dealing with the sideeffects causing concern to the patient and addressing any misconceptions about the treatment regime before commenting on other complications helps collaboration because patients see that their concerns are validated and given prominence. Such discussions may expose more significant problems in the coherence between the individual's model of the illness and the treatment advised. These can be explored through the cognitive techniques outlined in Box 2.

\section{Additional interventions for intentional non-adherers}

Interventions for intentional non-adherers initially target patients' understanding of the disorder and its treatment. Factors influencing their cognitive representation and perception of threat need to be understood. Also, the benefits of treatment need to be reinforced while barriers are modified or undermined (Scott \& Wright, 1997).

The first intervention provides patients with a realistic appraisal of the disorder and their prognosis. In some circumstances, this will take the form of an educational session supplemented by reading or video material to take home. An alternative, and often worthwhile, approach is to ask patients to take the first step and to seek information about the disorder as a homework assignment. A number of organisations produce booklets, leaflets and audiovisual aids that can be easily accessed. The clinician needs to make a judgement about whether this approach is positive, helpful or within the capability of a particular individual. However, with interested patients, it can be enlightening to get them to record their hypothesis about the aetiology of the disorder and to rate the percentage belief in the statement written. Patients are then encouraged to explore all sources of information and to write additional questions to bring to the next consultation. After discussing the accumulated evidence, they are asked to re-rate their belief in their original statement and/or to record their new hypothesis.

The second strategy is to explore the individual's pattern of non-adherence. This can be done through diary-keeping. Behavioural strategies may be employed to enhance adherence, but the information is mainly used to identify specific situations where there is a high risk of forgetting or omitting to take medication. When possible, negative automatic thoughts are noted (e.g. "If I take the lithium before the weekly management meeting, I'll be a zombie and make a fool of myself") and then explored within the consultation. Again, the emphasis is on guiding patients' examination of their cognitions. Alternative explanations and ratings of degree of belief in the thoughts need to be generated by the patient rather than the doctor. Experiments (e.g. asking patients to evaluate their performance in a meeting when medication is taken rather than omitted) can provide further data for such discussions.

Scott \& Wright (1997) also advocate the use of a cost-benefit analysis approach (see Box 3 ). With guidance from the doctor, the patient draws up a

Box 2. Interventions to enhance adherence

Behavioural strategies

Simplify regime

Behavioural prompts

Reinforcement strategies

Rehearsal

Monitoring and diary-keeping
Cognitive strategies

Homework assignments to develop realistic appraisal of prognosis

Identifying and challenging negative automatic thoughts regarding medication and illness

Identifying and developing an action plan to deal with high-risk situations

Re-framing or modifying underlying beliefs 
table outlining the advantages and disadvantages of taking medication, and the advantages and disadvantages of not taking medication. Patients add statements to each box, but the clinician tries to draw attention particularly to the advantages of adherence and the disadvantages of non-adherence in relation to patients' current and future life goals. If such discussions prove difficult, seeking a 'third party opinion' from someone else whom the patient trusts may also be useful. It helps patients retain trust in the clinician and ensures that they can continue their dialogue about medication adherence. Alternatively, significant others may be invited to attend sessions if their dysfunctional beliefs about the illness or its treatment are impeding the patient's adherence.

Patients' underlying beliefs may become apparent from the recurring themes in their negative automatic thoughts, the behaviours they engage in and the attitudes they express. Common themes related to non-adherence are autonomy and control. Although a detailed description of cognitive-behavioural interventions with dysfunctional beliefs is beyond the scope of this paper, the principles of interventions parallel those employed when exploring automatic thoughts. Modification of perfectionistic or control beliefs can be achieved through cognitive restructuring and behavioural experiments (e.g. noting exceptions in one's daily life to perfect behaviour or total control of situations). Alternatively, it may be possible to re-frame beliefs so that they operate to enhance rather than reduce adherence. For example, a patient believed that by trying harder, he or she could control the illness on his or her own. This often led to the patient stopping medication, but also to experiencing considerable knocks to his or her self-esteem when, despite best efforts, symptoms began to recur. Discussions focused on how the concept of 'trying harder' could incorporate independent and reasoned decisions about adherence. For example, trying harder to achieve one's life goals and to control the disorder could involve developing a greater understanding of the disorder and evidence for effective treatments, developing foolproof methods for maintaining adherence, and recognising and managing early warning signs of relapse.

In some cases, the doctor and patient have to 'agree to disagree' about the benefits of a particular treatment. However, clinicians need to ask themselves whether they are ignoring opportunities to make the regime more flexible, and/or whether the patient can be offered greater autonomy and control via a programme of self-management. The success of self-management for diabetes and asthma has led to a number of patient organisations exploring its use with mental disorders. For example, the Manic Depression Fellowship has produced a booklet that is quite compatible with the principles and practice of clinical psychiatry (e.g. teaching patients to recognise and monitor prodromal symptoms and proactively designing crisis intervention packages).

Sometimes it is a struggle to maintain any degree of engagement or collaboration with the patient. However, it is still more productive to try to work within a cognitive-behavioural framework and set up a collaborative experiment. If you are confident that patients will continue to attend appointments, the only realistic compromise may be a trial of treatment followed by a drug-free 'holiday' with

Box 3. Cost-benefit analysis of taking lithium

Advantages of taking lithium

Treatment keeps me out of the hospital

My family are less worried when I'm on lithium

I know I'm doing everything I can to keep

my illness under control

Disdvantages of taking lithium

I hate blood tests

I've gained weight as a side-effect

Lithium can be toxic and you can get

irreversible kidney damage

If my wife finds out, she'll be upset
Advantages of not taking lithium

I have fewer things to carry around and fewer

things to remember

$I^{\prime} m$ in control of me, not the tablets

Disavantages of not taking lithium

There is a greater risk I'll have a relapse

I might have to go back into hospital, and that might jeopardise my career

The doctor has expressed concern for my wellbeing if I don't use medication

Once, when depressed, I thought of killing myself - it was a frightening experience that I'd rather not have happen again 
continuous monitoring of symptoms throughout and increased frequency of clinical contact. This is not without drawbacks as, for example, relapse rates following lithium withdrawal are high. However, given a 'no win' situation, supervised reductions in medication and careful monitoring for early warning signs of relapse may be more acceptable than patients 'voting with their feet' and leaving the service, only to return some time later as involuntary admissions.

\section{Conclusions}

The practice of community psychiatry has highlighted that medication adherence is a rate-limiting step for maintaining people with severe mental disorders outside hospital. Before offering unequivocal support for the view that the widespread use of newer, more expensive medications will solve this problem, we need to explore the psychological, as opposed to the practical, problems that increase individuals' risk of non-adherence. Providing information to patients is an important strategy, but on its own is not enough. This paper suggests that greater emphasis should be given to the topic of adherence in psychiatric consultations. It also advocates a shift in consultation style towards a collaborative approach to treatment planning.

Many of the behavioural techniques noted represent an extension of the interventions most clinicians have used at some time. The modifications proposed are that the use of each technique is monitored and the outcomes noted, and that the overall strategy for understanding and enhancing adherence is more transparent and systematic. The additional advantage of actively discussing adherence with patients is that it often engages their curiosity and encourages them to review their model of their illness.

The cognitive techniques outlined extend beyond what many psychiatrists feel able to offer in routine practice. However, the importance of enhancing adherence may warrant the more widespread use of adherence or compliance therapies. Not all patients will be able to engage in these approaches, but Kemp et al (1996) and others have shown that compliance therapy can benefit patients with severe psychotic disorders. Furthermore, research with patients with bipolar disorders suggests that the cost of hospitalisation, and of medications that were prescribed but not taken, dramatically exceeds the cost of six hours of adherence therapy with a clinical psychologist or community psychiatric nurse, even when the cost of training and supervision is included (further details available from the author upon request). In the future, it may be possible to select individuals for this approach as an alternative to referral for a formal course of cognitive, family or other brief therapy.

\section{References}

Adams, J. \& Scott, J. (1999) Predicting medication nonadherence in severe mental disorders. Acta Psychiatrica Scandinavica, in press.

Becker, M. H. \& Maimon, L. A. (1975) Sociobehavioural determinants of compliance with health and medical care recommendations. Medical Care, 13, 10-24.

Buchanan, A. (1996) Compliance with Treatment in Schizophrenia. Maudsley Monographs. Hove: Psychology Press.

Cochran, S. (1984) Preventing medication non-compliance in the outpatient treatment of bipolar affective disorder. Journal of Nervous and Mental Diseases, 176, 457-464.

Dickson, W. E. \& Kendell, R. E. (1986) Does maintenance lithium therapy prevent recurrence of mania under ordinary clinical conditions? Psychological Medicine, 16, 521-530.

Goodwin, F. \& Jamison, K. (1990) Manic Depressive Illness. Oxford: Oxford University Press.

Haynes, B., Taylor, D. \& Sackett, D. (1979) Compliance in Health Care. Baltimore, MD: Johns Hopkins Press.

Horne, R. (1997) Representations of medication and treatment: advances in theory and measurement. In Perceptions of Health and Illness (eds K. J. Petrie \& J. Weinman), pp. 155-188. Amsterdam: Harwood Academic.

- \& Weinman, J. (1998) Predicting treatment adherence: An overview of theoretical models. In Adherence to Treatment in Medical Conditions (eds L. Myers and K. Midence), Pp. 113-132. Amsterdam: Harwood Academic.

Katon, W., von Korff, M., Lin, E., et al (1995) Collaborative management to achieve treatment guidelines. Journal of American Medical Association, 273, 1026-1031.

Keck, P., McElroy, B., Strakowski, S., et al (1998) Factors associated with pharmacologic non-compliance in patients with mania. Journal of Clinical Psychiatry, 57, 292-297.

Kemp, R., Hayward, P., Applewhaite, G., et al (1996) Compliance therapy in psychotic patients: randomized controlled trial. British Medical Journal, 312, 345-349.

Lin, E., von Korff, M., Katon, W., et al (1995) The role of the primary care physician in patients' adherence to antidepressant therapy. Medical Care, 33, 67-74.

Myers, E. D. \& Branthwaite, A. (1992) Outpatient compliance with antidepressant medication. British Journal of Psychiatry, 160, 83-86.

Scott, J. (1995) Psychotherapy for bipolar disorder. British Journal of Psychiatry, 167, 581-588.

- \& Wright, J. (1997) Cognitive therapy for severe mental disorders. In American Psychiatric Association Review of Psychiatry, Vol. 16, pp. 171-199. Washington DC: APA Press.

Skelton, J. \& Croyle, R. (1991) Mental Representation in Health and Illness. New York: Springer Verlag.

Stephenson, B. J., Rowe, B. H., Haynes, R. B., et al (1993) Is this patient taking the treatment as prescribed? Journal of the American Medical Association, 269, 2779-2781.

Weinstein, N. D. (1988) The precaution adoption process. Health Psychology, 7, 355-386.

Weintraub, M. (1990) Compliance in the elderly. Clinics in Geriatric Medicine, 6, 445-452.

Wright, E. (1993) Non-compliance - or how many aunts has Matilda? Lancet, 342, 909-913. 


\section{Multiple choice questions}

1. With regard to medication adherence:

a the 12-month relapse rate in depressed people who are non-adherent is over $75 \%$

b the 12-month relapse rate in people with schizophrenia who are non-adherent is greater than $50 \%$

c up to $60 \%$ of people hospitalised with bipolar disorder were non-adherent with medication in the month prior to admission

$\mathrm{d}$ the prevalence of non-adherence in people with severe mental disorders has fallen in the past 20 years

e people who are unintentional non-adherers are also known as 'intelligent non-adherers'.

2. Key components of the cognitive representation of illness are:
a time-line
b susceptibility
c cure
d identity
e consequences.

3. Key beliefs that contribute the health belief model are :

a perceived severity

b perceived benefits of treatment

c perceived threat

d perceived susceptibility

e perceived barriers to treatment.
4. Patients are more likely to be adherent to a treatment regime if:

a it is acceptable

b it is understandable

c if it is manageable

d there is coherence between patients' abstract ideas about the illness and their concrete experience of symptoms

e there is coherence between their cognitive representation of the illness and the psychiatrist's instructions.

5. Techniques that have been shown to enhance medication adherence are:

a behavioural reinforcement

b having a 'straight talk' with patients about their non-adherence

c identifying and challenging negative automatic thoughts about their illness

$d$ ensuring that the perceived benefits of adherence exceed the perceived barriers

e reframing underlying beliefs.

MCQ answers

$\begin{array}{llllllll}\text { 1 } & & \text { 2 } & & 3 & 4 & 5 \\ \text { a T } & \text { a } & \text { T } & \text { a } & \text { T } & \text { a } & \text { T } & \text { a T } \\ \text { b T } & \text { b F } & \text { b T } & \text { b T } & \text { b T } \\ \text { c T } & \text { c T } & \text { c T } & \text { c T } & \text { c T } \\ \text { d F } & \text { d T } & \text { d T } & \text { d T } & \text { d T } \\ \text { e F } & \text { e T } & \text { e T } & \text { e T } & \text { e T }\end{array}$

\section{Commentary}

\section{Amanda Harris}

Professor Scott sums up the views of many of the Manic Depression Fellowship's (MDF) members when she advocates a shift in consultation style towards a collaborative approach to treatment planning.
A survey of the membership undertaken in association with the Sainsbury Centre for Mental Health (Hill et al, 1996) and our ongoing evaluation of our Self Management Programme have both highlighted this issue. We now have a good deal of

Amanda Harris works as the National Self Managemeht Programme Manager for the Manic Depression Fellowship (8-10 High Street, Kingston upon Thames, Surrey KT11 1EY). She is undertaking research in management and organisational learning, and the integration of an evidence-based approach with continuing professional development and knowledge management. 